ISSN 1855-3966 (printed edn.), ISSN 1855-3974 (electronic edn.)

ARS MATHEMATICA CONTEMPORANEA 16 (2019) 359-375

https://doi.org/10.26493/1855-3974.1525.7f3

(Also available at http://amc-journal.eu)

\title{
A characterization of graphs with disjoint total dominating sets*
}

\author{
Michael A. Henning ${ }^{\dagger}$ \\ Department of Pure and Applied Mathematics, University of Johannesburg, \\ Auckland Park, 2006 South Africa \\ Iztok Peterin $\ddagger$ \\ Faculty of Electrical Engineering and Computer Science, University of Maribor, \\ Koroška 46, 2000 Maribor, Slovenia
}

Received 8 November 2017, accepted 11 November 2018, published online 27 January 2019

\begin{abstract}
A set $S$ of vertices in a graph $G$ is a total dominating set of $G$ if every vertex is adjacent to a vertex in $S$. A fundamental problem in total domination theory in graphs is to determine which graphs have two disjoint total dominating sets. In this paper, we solve this problem by providing a constructive characterization of the graphs that have two disjoint total dominating sets. Our characterization gives an entirely new description of graphs with two disjoint total dominating sets and places them in another context, developing them from four base graphs and applies a sequence of operations from seventeen operations that are independent and necessary to produce all such graphs. We show that every graph with two disjoint total dominating sets can be constructed using this method.
\end{abstract}

Keywords: Total domination number, disjoint total dominating sets.

Math. Subj. Class.: 05C69

\footnotetext{
*The authors express their sincere thanks to the referees for their meticulous and thorough reading of the paper, and for their very helpful comments which improved the exposition and clarity of the revised version of the paper. In particular, we thank one of the reviewers for suggesting to us Claim 4.9 and Claim 4.10 which greatly simplified the original proof.

${ }^{\dagger}$ Research supported in part by the South African National Research Foundation and the University of Johannesburg.

¥This author is also affiliated with Institute of Mathematics, Physics and Mechanics, Jadranska 19, 1000 Ljubljana, Slovenia. Research supported in part by the Slovenian Research Agency by the projects No. P1-0297 and No. J1-9109.

E-mail addresses: mahenning@uj.ac.za (Michael A. Henning), iztok.peterin@um.si (Iztok Peterin)
}

()(7) This work is licensed under https://creativecommons.org/licenses/by/4.0/ 


\section{Introduction}

A dominating set of a graph $G$ is a set $S$ of vertices of $G$ such that every vertex not in $S$ has a neighbor in $S$, where two vertices are neighbors if they are adjacent. A total dominating set of a graph $G$ with no isolated vertex is a set $S$ of vertices such that every vertex in $G$ has a neighbor in $S$. Domination and its variations in graphs are now well studied. The literature on this subject has been surveyed and detailed in the two books by Haynes, Hedetniemi, and Slater [6, 7]. For a recent book on total domination in graphs we refer the reader to [13]. A survey of total domination in graphs can also be found in [9].

A classical result in domination theory, due to Ore [14] in 1962, is that every graph with no isolated vertex has two disjoint dominating sets. However, it is not the case that every graph with no isolated vertex can be partitioned into a dominating set and a total dominating set. Henning and Southey [11] showed that every connected graph with minimum degree at least two that is not a cycle on five vertices has a disjoint dominating set and a total dominating set. Further, in [12] they present a constructive characterization of connected graphs of order at least 4 that have a disjoint dominating set and a total dominating set. Disjoint dominating and total dominating sets in graphs are studied further, for example, in [10]. A characterization of graphs with disjoint dominating and paired-dominating sets is characterized in [15].

It remains, however, an outstanding problem to determine which graphs have two disjoint total dominating sets. Zelinka [16] in 1989 showed that no minimum degree condition in a graph is sufficient to guarantee that there exist two disjoint total dominating sets in the graph. Heggernes and Telle [8] showed that the decision problem to decide for a given graph $G$ if it has two disjoint total dominating sets is NP-complete, even for bipartite graphs. Sufficient conditions for a graph to have two disjoint total dominating sets were obtained by Delgado, Desormeaux, and Haynes [4], but the authors in [4] were not able to characterize such graphs. Cubic graphs that have two disjoint total dominating sets were recently studied by Desormeaux, Henning and Haynes [5]. In particular, they show that cubic graphs that are 5 -chordal or claw-free (we do not define these concepts here) can be partitioned into two total dominating sets.

The total domatic number $\operatorname{tdom}(G)$ of $G$ is the maximum number of disjoint total dominating sets [3]. This can also be considered as a coloring of the vertices such that every vertex has a neighbor of every color (and has been called the coupon coloring problem [2]). Recent work on the total domatic number can be found, for example, in [1,5]. The fundamental problem in total domination theory in graphs of determining which graphs have two disjoint total dominating sets can be phrased as follows: Determine which graphs $G$ satisfy $\operatorname{tdom}(G) \geq 2$. We call a graph a TDP-graph (standing for "total dominating partitionable graph") if its vertex set can be partitioned into two total dominating sets; that is, a graph $G$ is a TDP-graph if and only if $\operatorname{tdom}(G) \geq 2$.

In this paper, we provide a constructive characterization of the graphs that have two disjoint total dominating sets, or, equivalently, a characterization of the TDP-graphs. We describe a procedure to build TDP-graphs in terms of a 2-coloring of the vertices that indicate the role each vertex plays in the sets associated with the two disjoint total dominating sets. We show that the resulting family we construct, starting from four initial base graphs and applying one of seventeen operations to extend graphs in the family to larger graphs, is precisely the class of all TDP-graphs.

Our characterization provides a method for creating the TDP-graphs using a finite set of precise operations. The construction places the TDP-graphs in another context, devel- 
oping them from four base graphs and applying a sequence of operations from seventeen operations that are independent and necessary to produce a TDP-graph; that is, we show that this method produces precisely the family of TDP-graphs in that every graph generated by this method/algorithm is a TDP-graph and further every TDP-graph can be constructed in this way.

We remark that this procedure does not solve the decision problem to decide if a given graph has two disjoint total dominating sets in polynomial time. If one follows the steps in the proof of Section 4, one does indeed obtain an algorithm for this decision problem. However, this algorithm is far from polynomial time complexity. In particular, the first step of this algorithm is to discard some edges in order to obtain so-called sparse TDPgraph. Unfortunately, the proof does not provide those edges and this already spoils the time complexity.

\subsection{Notation}

For notation and graph theory terminology we generally follow [13]. All graphs in this paper are finite and simple, without loops or multiple edges. The order of a graph $G$ is denoted by $n(G)=|V(G)|$, and the size of $G$ by $m(G)=|E(G)|$. We denote the degree of a vertex $v$ in the graph $G$ by $d_{G}(v)$. The maximum (minimum) degree among the vertices of $G$ is denoted by $\Delta(G)(\delta(G)$, respectively). The open neighborhood of $v$ is $N_{G}(v)=\{u \in V(G) \mid u v \in E(G)\}$. For a set $S \subseteq V(G)$, its open neighborhood is the set $N_{G}(S)=\bigcup_{v \in S} N_{G}(v)$. For subsets $X$ and $Y$ of vertices of $G$, we denote the set of edges with one end in $X$ and the other end in $Y$ by $[X, Y]$. For a set $S \subseteq V(G)$, the subgraph induced by $S$ is denoted by $G[S]$. Further, the subgraph of $G$ obtained from $G$ by deleting all vertices in $S$ and all edges incident with vertices in $S$ is denoted by $G-S$; that is, $G-S=G[V(G) \backslash S]$. If $S=\{v\}$, we simply denote $G-\{v\}$ by $G-v$.

The distance between two vertices $u$ and $v$ in $G$, denoted $d_{G}(u, v)$, is the minimum length of a $(u, v)$-path in $G$. By $W_{u v}$ we denote the set of all vertices of $G$ which are closer to $u$ than to $v$; that is, $W_{u v}=\left\{w \mid d_{G}(w, u)<d_{G}(w, v)\right\}$. Symmetrically, $W_{v u}$ is defined. A block of a graph $G$ is a maximal connected subgraph of $G$ which has no cut-vertex of its own. A block containing exactly one cut-vertex of $G$ is called an end-block. It is well known that any two different blocks of a graph have at most one vertex in common, namely a cut-vertex. Furthermore, a connected graph with at least one cut-vertex has at least two end-blocks. Let $X$ denote the set of cut-vertices of a connected graph $G$ and let $Y$ denote the set of its blocks. The block graph of $G$ is a bipartite graph with partite sets $X$ and $Y$ in which a vertex $x \in X$ is adjacent to a vertex $y \in Y$ if $x$ is a vertex of the block $y$. It is well-known that the block graph of any connected graph is a tree.

A walk is a finite, alternating sequence of vertices and edges in which each edge of the sequence joins the vertex that precedes it in the sequence to the vertex that follows it in the sequence. A non-backtracking walk is a walk with the additional constraint that no two consecutive edges on the walk are repeated.

Let $u$ be a cut-vertex of a graph $G$. Let $H_{1}$ and $H_{2}$ be two vertex disjoint subgraphs of $G-u$ that contain all the components of $G-u$, where each of $H_{1}$ and $H_{2}$ contain at least one component of $G-u$. We call $H_{1}$ and $H_{2}$ the associated subgraphs of $G-u$. For $i \in[2]$, we denote by $H_{i}^{u}$ the subgraph of $G$ induced by $V\left(H_{i}\right) \cup\{u\}$. Further, the vertex in $H_{1}^{u}$ named $u$ we rename $u^{\prime}$, and the vertex in $H_{2}^{u}$ named $u$ we rename $u^{\prime \prime}$ in order to distinguish between $u, u^{\prime}$ and $u^{\prime \prime}$. We use the standard notation $[k]=\{1, \ldots, k\}$. 


\section{The graph family $\mathcal{G}$}

In this section, we construct a graph family $\mathcal{G}$ such that every graph in the family has two disjoint total dominating sets. First, we define a 2-coloring of a graph $G$ as a partition $S=$ $\left(S_{R}, S_{B}\right)$ of $V(G)$. The color of a vertex $v$, denoted $\operatorname{color}(v)$, is the letter $X \in\{R, B\}$ such that $v \in S_{X}$, where " $R$ " and " $B$ " here stand for red and blue, respectively. Thus, our 2-coloring of $G$ is a coloring of the vertices of $G$, one color to each vertex, using the colors red and blue. We denote by $\bar{X}$ the letter $\bar{X} \in\{R, B\} \backslash\{X\}$, and we call $\bar{X}$ the color different from $X$. Thus, if $X=R$, then $\bar{X}=B$ while if $X=B$, then $\bar{X}=R$. We denote by $(G, S)$ a graph $G$ with a given 2-coloring $S$. Our aim is to describe a procedure to build TDP-graphs in terms of 2-colorings. For $i \in[4]$, by a 2 -colored $G_{i}$, we shall mean the graph $G_{i}$ and its associated 2-coloring shown in Figure 1. Further, we call each 2-colored $G_{i}$ a 2 -colored base graph.

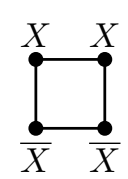

(a) $G_{1}$

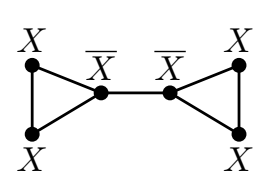

(b) $G_{2}$

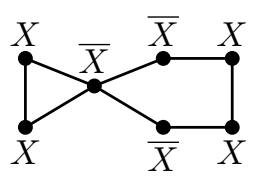

(c) $G_{3}$

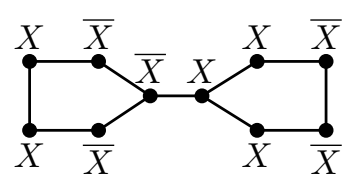

(d) $G_{4}$

Figure 1: The four 2-colored base graphs $G_{1}, G_{2}, G_{3}, G_{4}$.

Let $\mathcal{G}$ be the minimum family of 2 -colored graphs that:

(i) contains the four 2-colored base graphs; and

(ii) is closed under the seventeen operations $\mathcal{O}_{1}$ through to $\mathcal{O}_{17}$ listed below, which extend a 2-colored graph $\left(G^{\prime}, S^{\prime}\right)$ to a new 2-colored graph $(G, S)$.

In Figures $2-7$, the vertices of $G^{\prime}$ are colored black and the new vertices of $G$ are colored white for illustrative purposes, even though the actual colors of the vertices are indicated by the letters $X$ and $\bar{X}$.

Operation $\mathcal{O}_{1}:(G, S)$ is obtained from $\left(G^{\prime}, S^{\prime}\right)$ by adding an edge between two nonadjacent vertices of the same color. See the upper diagram of Figure 2.

Operation $\mathcal{O}_{2}:(G, S)$ is obtained from $\left(G^{\prime}, S^{\prime}\right)$ by adding an edge between two nonadjacent vertices of different color. See the lower diagram of Figure 2.

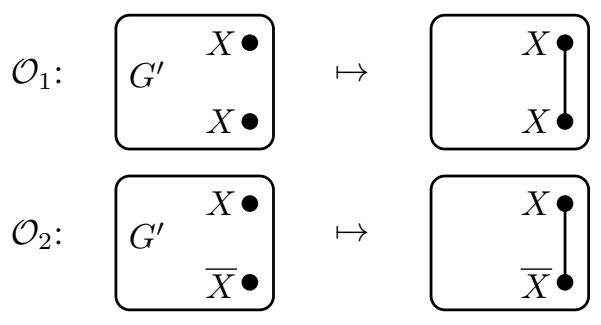

Figure 2: The operations $\mathcal{O}_{1}$ and $\mathcal{O}_{2}$. 
Operation $\mathcal{O}_{3}$ : If $u$ and $v$ are distinct vertices of different color from $\left(G^{\prime}, S^{\prime}\right)$, then $(G, S)$ is obtained from $\left(G^{\prime}, S^{\prime}\right)$ by adding a new vertex of any color adjacent to both $u$ and $v$. See the left diagram in the upper part of Figure 3.

Operation $\mathcal{O}_{4}$ : If $u$ and $v$ are distinct vertices of the same color from $\left(G^{\prime}, S^{\prime}\right)$, then $(G, S)$ is obtained from $\left(G^{\prime}, S^{\prime}\right)$ by adding adjacent vertices $x$ and $y$ and edges $u x$ and $v y$ with $\operatorname{color}(x)=\operatorname{color}(y) \neq \operatorname{color}(u)$. See the middle diagram in the upper part of Figure 3 .

Operation $\mathcal{O}_{5}$ : If $u$ and $v$ are distinct vertices of different color from $\left(G^{\prime}, S^{\prime}\right)$, then $(G, S)$ is obtained from $\left(G^{\prime}, S^{\prime}\right)$ by adding adjacent vertices $x$ and $y$ and edges $u x$ and $v y$ with $\operatorname{color}(x)=\operatorname{color}(u) \neq \operatorname{color}(y)$. See the right diagram in the upper part of Figure 3.

Operation $\mathcal{O}_{6}$ : If $u$ and $v$ are distinct vertices of the same color from $\left(G^{\prime}, S^{\prime}\right)$, then $(G, S)$ is obtained from $\left(G^{\prime}, S^{\prime}\right)$ by adding a path $x y z$ with $\operatorname{color}(y)=\operatorname{color}(z) \neq \operatorname{color}(x)=$ color $(u)$ and adding edges $u x$ and $v z$. See the left diagram in the lower part of Figure 3.

Operation $\mathcal{O}_{7}$ : If $u$ and $v$ are distinct vertices of the same color from $\left(G^{\prime}, S^{\prime}\right)$, then $(G, S)$ is obtained from $\left(G^{\prime}, S^{\prime}\right)$ by adding a path $x y z w$ and edges $u x$ and $v w$ with $\operatorname{color}(x)=$ $\operatorname{color}(w)=\operatorname{color}(u) \neq \operatorname{color}(y)=\operatorname{color}(z)$. See the middle diagram in the lower part of Figure 3.
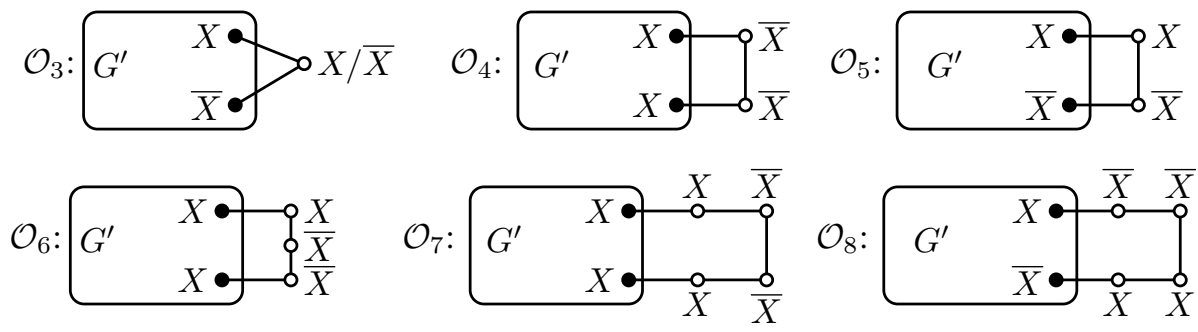

Figure 3: The operations $\mathcal{O}_{3}-\mathcal{O}_{8}$.

Operation $\mathcal{O}_{8}$ : If $u$ and $v$ are distinct vertices of different color from $\left(G^{\prime}, S^{\prime}\right)$, then $(G, S)$ is obtained from $\left(G^{\prime}, S^{\prime}\right)$ by adding a path $x y z w$ and edges $u x$ and $v w$ with $\operatorname{color}(x)=$ $\operatorname{color}(y)=\operatorname{color}(v) \neq \operatorname{color}(z)=\operatorname{color}(w)$. See the right diagram in the lower part of Figure 3.

Operation $\mathcal{O}_{9}$ : If $u$ and $v$ are adjacent vertices of different color from $\left(G^{\prime}, S^{\prime}\right)$, then $(G, S)$ is obtained from $\left(G^{\prime}, S^{\prime}\right)$ by subdividing $u v$ with four consecutive vertices $x, y, z, w$ where $x$ is adjacent to $u$ and $\operatorname{color}(u)=\operatorname{color}(z)=\operatorname{color}(w) \neq \operatorname{color}(x)=\operatorname{color}(y)$. See the upper diagram of Figure 4.

Operation $\mathcal{O}_{10}$ : If $u$ and $v$ are adjacent vertices of the same color from $\left(G^{\prime}, S^{\prime}\right)$, then $(G, S)$ is obtained from $\left(G^{\prime}, S^{\prime}\right)$ by subdividing $u v$ with four consecutive vertices $x, y, z, w$ where $x$ is adjacent to $u$ and $\operatorname{color}(u)=\operatorname{color}(x)=\operatorname{color}(w) \neq \operatorname{color}(y)=\operatorname{color}(z)$. See the lower diagram of Figure 4.

Operation $\mathcal{O}_{11}$ : If $v$ is a vertex from $\left(G^{\prime}, S^{\prime}\right)$, then $(G, S)$ is obtained from $\left(G^{\prime}, S^{\prime}\right)$ by adding an edge $x y$ together with the edges $v x$ and $v y$ where $\operatorname{color}(x)=\operatorname{color}(y) \neq$ $\operatorname{color}(v)$. See the left diagram of Figure 5.

Operation $\mathcal{O}_{12}$ : If $v$ is a vertex from $\left(G^{\prime}, S^{\prime}\right)$, then $(G, S)$ is obtained from $\left(G^{\prime}, S^{\prime}\right)$ by 


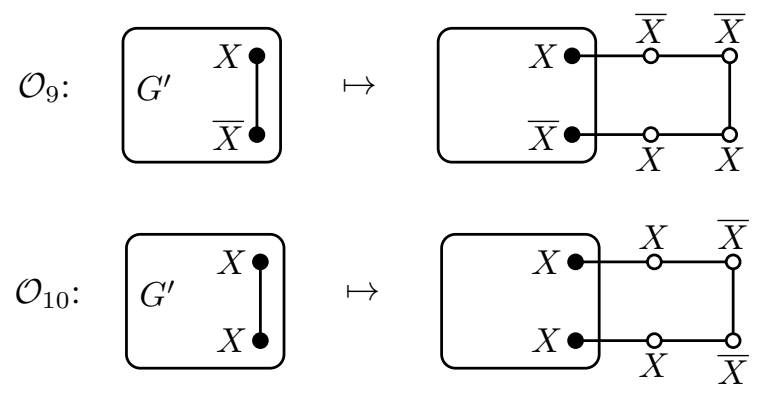

Figure 4: The operations $\mathcal{O}_{9}$ and $\mathcal{O}_{10}$.

adding a path $x y z$ together with the edges $v x$ and $v z$ where $\operatorname{color}(x)=\operatorname{color}(y) \neq$ $\operatorname{color}(z)=\operatorname{color}(v)$. See the middle diagram of Figure 5 .

Operation $\mathcal{O}_{13}$ : If $v$ is a vertex from $\left(G^{\prime}, S^{\prime}\right)$, then $(G, S)$ is obtained from $\left(G^{\prime}, S^{\prime}\right)$ by adding a path $x y z w$ together with the edges $v x$ and $v w$ where $\operatorname{color}(x)=\operatorname{color}(w)=$ $\operatorname{color}(v) \neq \operatorname{color}(y)=\operatorname{color}(z)$. See the right diagram of Figure 5.
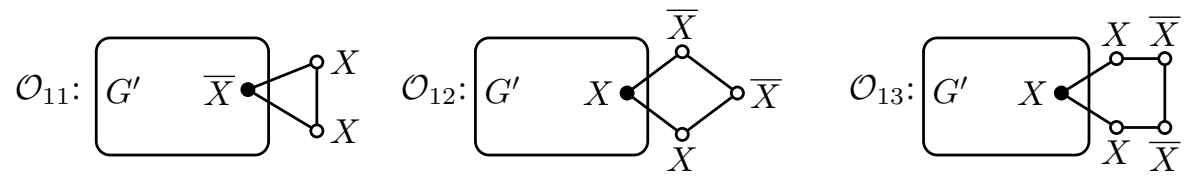

Figure 5: The operations $\mathcal{O}_{11}, \mathcal{O}_{12}$ and $\mathcal{O}_{13}$.

Operation $\mathcal{O}_{14}$ : If $v$ is a vertex from $\left(G^{\prime}, S^{\prime}\right)$, then $(G, S)$ is obtained from $\left(G^{\prime}, S^{\prime}\right)$ by adding a 3-cycle, $x y z x$, together with the edge $v x$ where $\operatorname{color}(x)=\operatorname{color}(v) \neq$ $\operatorname{color}(y)=\operatorname{color}(z)$. See the left diagram of Figure 6 .

Operation $\mathcal{O}_{15}$ : If $v$ is a vertex from $\left(G^{\prime}, S^{\prime}\right)$ of any color, then $(G, S)$ is obtained from $\left(G^{\prime}, S^{\prime}\right)$ by adding a 4-cycle, $x y z w x$, together with the edge $v x$ where $\operatorname{color}(x)=\operatorname{color}(y) \neq$ $\operatorname{color}(z)=\operatorname{color}(w)$. See the middle diagram of Figure 6 , where the notation $X / \bar{X}$ means that the vertex can have any color.

Operation $\mathcal{O}_{16}$ : If $v$ is a vertex from $\left(G^{\prime}, S^{\prime}\right)$, then $(G, S)$ is obtained from $\left(G^{\prime}, S^{\prime}\right)$ by adding a 5-cycle, $x y z w t x$, together with the edge $v x$ where $\operatorname{color}(x)=\operatorname{color}(y)=$ $\operatorname{color}(t) \neq \operatorname{color}(z)=\operatorname{color}(w)=\operatorname{color}(v)$. See the right diagram of Figure 6.

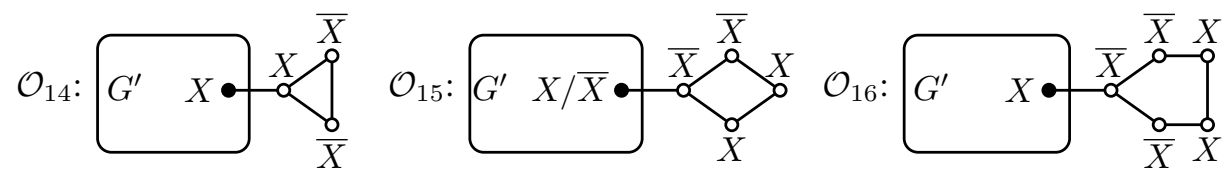

Figure 6: The operations $\mathcal{O}_{14}, \mathcal{O}_{15}$ and $\mathcal{O}_{16}$.

Operation $\mathcal{O}_{17}$ : If $u$ is a cut-vertex from $\left(G^{\prime}, S^{\prime}\right)$ with associated subgraphs $H_{1}^{u}$ and $H_{2}^{u}$, 
and in $N_{H_{1}^{u}}\left(u^{\prime}\right)$ there exists a vertex of the same color as $u$ and in $N_{H_{2}^{u}}\left(u^{\prime \prime}\right)$ there exists a vertex of different color as $u$, then $(G, S)$ is obtained from $H_{1}^{u}$ and $H_{2}^{u}$ by adding a new vertex $v$ and the edges $u^{\prime} v$ and $v u^{\prime \prime}$. The color of all vertices from $H_{1}^{u}$ remains the same as in $G^{\prime}, \operatorname{color}(v)=\operatorname{color}\left(u^{\prime \prime}\right) \neq \operatorname{color}\left(u^{\prime}\right)=\operatorname{color}(u)$ and the color of all vertices from $H_{2}^{u}$ is exchanged with respect to their color in $G^{\prime}$. See the diagram of Figure 7, where the notation $\bar{A}$ means that the color of all vertices from the set $A$ in $\left(G^{\prime}, S^{\prime}\right)$ is changed in $(G, S)$.

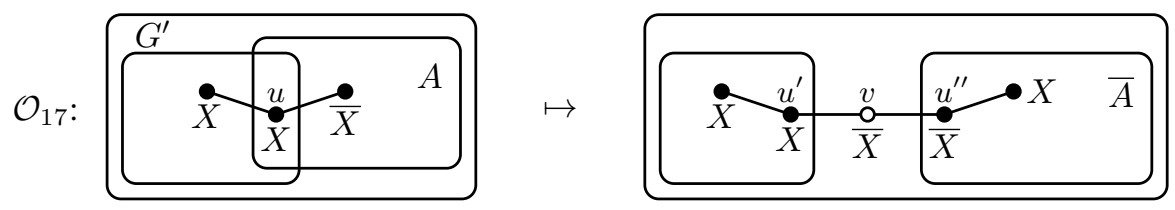

Figure 7: The operation $\mathcal{O}_{17}$.

We remark that, by definition, all operations $\mathcal{O}_{3}$ to $\mathcal{O}_{17}$ produce new vertices. Further, exactly one new vertex created in each of the operations $\mathcal{O}_{14}$ to $\mathcal{O}_{16}$ has degree 3 , and all other new vertices created using operations $\mathcal{O}_{3}$ to $\mathcal{O}_{17}$ have degree $2 \mathrm{in} G$. In operations $\mathcal{O}_{11}$ to $\mathcal{O}_{13}$, if the selected vertex $v$ from $\left(G^{\prime}, S^{\prime}\right)$ is a cut-vertex of $G^{\prime}$ it is also a cutvertex in $G$, while if $v$ is not a cut-vertex of $G^{\prime}$ it becomes a cut-vertex in $G$. Moreover all operations from $\mathcal{O}_{14}$ to $\mathcal{O}_{17}$ produce new cut vertices. In this sense all operations, except $\mathcal{O}_{1}$ and $\mathcal{O}_{2}$, can be viewed as base operations which build the sparse skeleton of TDPgraphs, while $\mathcal{O}_{1}$ and $\mathcal{O}_{2}$ fill this skeleton with additional edges. This is also the main idea of the proof. First to discard all edges which are there by one of the operations $\mathcal{O}_{1}$ and $\mathcal{O}_{2}$, and then study the resulting vertices of degree two.

Lemma 2.1. If $(G, S) \in \mathcal{G}$ for some 2-coloring $S=\left(S_{R}, S_{B}\right)$, then $G$ is a TDP-graph. Further, $S=\left(S_{R}, S_{B}\right)$ is a partition of $V(G)$ into two total dominating sets of $G$.

Proof. We proceed by induction on the number, $k \geq 0$, of operations $\mathcal{O}_{1}$ through $\mathcal{O}_{17}$ used to construct a 2-colored graph $(G, S) \in \mathcal{G}$. If $k=0$, then $(G, S)$ is one of the four 2 -colored base graphs illustrated in Figure 1, and one can readily observe that $G$ is a TDPgraph and $S=\left(S_{R}, S_{B}\right)$ is a partition of $V(G)$ into two total dominating sets of $G$. This establishes the base case. Let $k \geq 1$ and suppose that every 2-colored graph $\left(G^{\prime}, S^{\prime}\right) \in \mathcal{G}$ that can be constructed using fewer than $k$ operations satisfies the desired result.

Let $(G, S) \in \mathcal{G}$ be a 2-colored graph that can be built from one of the 2-colored base graphs by a sequence of $k$ operations $\mathcal{O}_{1}-\mathcal{O}_{17}$. Let $\mathcal{O}_{j}$ be the last operation of that sequence where $j \in[17]$, and let $\left(G^{\prime}, S^{\prime}\right)$ be the graph obtained from the same 2-colored base graph with the same sequence as that used to construct $(G, S)$ but without applying the last operation $\mathcal{O}_{j}$. Thus, $\left(G^{\prime}, S^{\prime}\right) \in \mathcal{G}$ can be constructed using fewer than $k$ operations.

By the induction hypothesis, the graph $G^{\prime}$ is a TDP-graph and $S^{\prime}=\left(S_{A}^{\prime}, S_{B}^{\prime}\right)$ is a partition of $V\left(G^{\prime}\right)$ into two total dominating sets of $G^{\prime}$. If $j \in[2]$, then $S=S^{\prime}$ and $G$ is a TDP-graph since no new vertices were added. For $3 \leq j \leq 17$ it is a simple exercise to check from the color of the new vertices added to $\left(G^{\prime}, S^{\prime}\right)$ when forming $(G, S)$ that the operation $\mathcal{O}_{j}$ yields two disjoint total domination sets, namely $S_{R}$ and $S_{B}$. Thus, $G$ is a TDP-graph, and $S=\left(S_{R}, S_{B}\right)$ is a partition of $V(G)$ into two total dominating sets of $G$. 


\section{Main result}

Our main result is to provide a constructive characterization of the graphs that have two disjoint total dominating sets, or, equivalently, a characterization of the TDP-graphs.

We prove that the class of all TDP-graphs is precisely the family $\mathcal{G}$ constructed in Section 2. A proof of Theorem 3.1 is given in Section 4.

Theorem 3.1. A graph $G$ is a TDP-graph if and only if every component of $(G, S)$ is in $\mathcal{G}$ for some 2-coloring $S$. Further, if $(G, S) \in \mathcal{G}$, then $S=\left(S_{R}, S_{B}\right)$ is a partition of $V(G)$ into two total dominating sets of $G$.

\section{Proof of Theorem 3.1}

The sufficiency follows from Lemma 2.1. To prove the necessity, let $G$ be a TDP-graph and let $S=\left(S_{R}, S_{B}\right)$ be a partition of $V(G)$ into two total dominating sets of $G$. We show that $(G, S) \in \mathcal{G}$ by induction on $m=|E(G)|$. Since $G$ is a TDP-graph, we note that $\delta(G) \geq 2, G$ has order $n \geq 4$, and $m \geq 4$. If $m=4$, then necessarily $G \cong C_{4}$, and $(G, S)$ is the 2 -colored base graph $G_{1}$, and so $(G, S) \in \mathcal{G}$. This establishes the base case. Let $m \geq 5$ and assume that every TDP-graph $G^{\prime}$ of size less than $m$ where $S^{\prime}=\left(S_{R}^{\prime}, S_{B}^{\prime}\right)$ is a partition of $V\left(G^{\prime}\right)$ into two total dominating sets satisfies $\left(G^{\prime}, S^{\prime}\right) \in \mathcal{G}$.

Let $G$ be a TDP-graph of order $n$ and size $m$, and let $S=\left(S_{R}, S_{B}\right)$ be a partition of $V(G)$ into two total dominating sets of $G$. If $G$ is disconnected, we apply the inductive hypothesis to each component of $G$ to produce the desired result. Hence, we may assume that $G$ is connected.

Our general strategy in what follows is to reduce the graph $G$ to a TDP-graph $G^{\prime}$ of size less than $m$, apply the inductive hypothesis to $G^{\prime}$ to show that $\left(G^{\prime}, S^{\prime}\right) \in \mathcal{G}$, and then reconstruct the graph $(G, S)$ from $\left(G^{\prime}, S^{\prime}\right)$ by applying one of the operations $\mathcal{O}_{x}, x \in[17]$, to show that $(G, S) \in \mathcal{G}$. We state this formally, since we will frequently use the following statement.

Statement 4.1. If $G^{\prime}$ is a TDP-graph of size less than $m$, where $S^{\prime}=\left(S_{R}^{\prime}, S_{B}^{\prime}\right)$ is a partition of $V\left(G^{\prime}\right)$ into two total dominating sets, and $(G, S)$ can be constructed from $\left(G^{\prime}, S^{\prime}\right)$ by applying one of the operations $\mathcal{O}_{x}$, where $x \in[17]$, then $(G, S) \in \mathcal{G}$.

We define three graphs $G_{R}, G_{B}$ and $G_{R B}$ associated with the graph $G$ and the partition $S=\left(S_{R}, S_{B}\right)$. Let $G_{R}$ and $G_{B}$ be the subgraphs of $G$ induced by the sets $S_{R}$ and $S_{B}$, respectively, and so $G_{R}=G\left[S_{R}\right]$ and $G_{B}=G\left[S_{B}\right]$. Let $G_{R B}$ be the (spanning) subgraph of $G$ with $V\left(G_{R B}\right)=V(G)$ and $E\left(G_{R B}\right)=E(G) \backslash\left(E\left(G_{R}\right) \cup E\left(G_{B}\right)\right)$.

Claim 4.2. If some component of $G_{R}, G_{B}$ or $G_{R B}$ is not a star, then $(G, S) \in \mathcal{G}$.

Proof. Suppose that there exists a component, $C$, of $G_{R}, G_{B}$ or $G_{R B}$ which is not a star. If $C$ contains a cycle $v_{1} \ldots v_{k} v_{1}, k \geq 3$, then $G$ can be obtained from $G^{\prime}=G-v_{1} v_{2}$ by either applying operation $\mathcal{O}_{1}$ in the case when $C$ is a component of $G_{R}$ or $G_{B}$ or by applying operation $\mathcal{O}_{2}$ in the case when $C$ is a component of $G_{R B}$. If $C$ contains no cycle, then $C$ is a tree different from a star. Therefore, there exists a path $u_{1} u_{2} u_{3} u_{4}$ in $C$ and $G$ can be obtained from $G^{\prime}=G-u_{2} u_{3}$ by either applying operation $\mathcal{O}_{1}$ in the case when $C$ is a component of $G_{R}$ or $G_{B}$ or by applying operation $\mathcal{O}_{2}$ in the case when $C$ is a component of $G_{R B}$. In all cases, since $S=\left(S_{R}, S_{B}\right)$ is a partition of $V(G)$ into two total dominating sets of $G$, the same partition $S^{\prime}=S=\left(S_{R}, S_{B}\right)$ is a partition of $V\left(G^{\prime}\right)$ into two total 
dominating sets of $G^{\prime}$. By the inductive hypothesis, $\left(G^{\prime}, S^{\prime}\right) \in \mathcal{G}$. We can obtain $G$ from the same 2-colored base graph as $G^{\prime}$ and the same sequence of operations from $\mathcal{O}_{1}-\mathcal{O}_{17}$ used to construct $\left(G^{\prime}, S^{\prime}\right)$ by adding at the end of this sequence the operation $\mathcal{O}_{1}$ or $\mathcal{O}_{2}$. Hence $(G, S) \in \mathcal{G}$.

By Claim 4.2, we may assume that every component of $G_{R}, G_{B}$ or $G_{R B}$ is a star, for otherwise the desired result follows. We call the resulting graph $G$ a sparse TDP-graph with associated partition $S=\left(S_{R}, S_{B}\right)$.

We now partition the sets $S_{R}$ and $S_{B}$ in two different ways depending on the role that the vertices in $S_{R}$ and $S_{B}$, respectively, play in the graphs $G_{R}, G_{B}$ and $G_{R B}$. First, let $S_{R}=R_{1} \cup R_{2} \cup R_{3}$ and $S_{B}=B_{1} \cup B_{2} \cup B_{3}$ where

$$
\begin{aligned}
& R_{1}=\left\{v \in S_{R} \mid d_{G_{R}}(v) \geq 2\right\} \\
& R_{2}=\left\{v \in S_{R} \backslash R_{1} \mid N_{G}(v) \cap R_{1} \neq \emptyset\right\} \\
& R_{3}=S_{R} \backslash\left(R_{1} \cup R_{2}\right)
\end{aligned}
$$

and

$$
\begin{aligned}
& B_{1}=\left\{v \in S_{B} \mid d_{G_{B}}(v) \geq 2\right\} \\
& B_{2}=\left\{v \in S_{B} \backslash B_{1} \mid N_{G}(v) \cap B_{1} \neq \emptyset\right\} \\
& B_{3}=S_{B} \backslash\left(B_{1} \cup B_{2}\right) .
\end{aligned}
$$

Next, we define a partition of $V(G)=V\left(G_{R B}\right)$ as the union of the two partitions $S_{R}=R_{1} B \cup R_{2} B \cup R_{3} B$ and $S_{B}=R B_{1} \cup R B_{2} \cup R B_{3}$ where

$$
\begin{aligned}
& R_{1} B=\left\{v \in S_{R} \mid d_{G_{R B}}(v) \geq 2\right\} \\
& R_{2} B=\left\{v \in S_{R} \backslash R_{1} B \mid v \text { has a neighbor in } G_{R B} \text { that belongs to } R B_{1}\right\} \\
& R_{3} B=S_{R} \backslash\left(R_{1} B \cup R_{2} B\right)
\end{aligned}
$$

and

$$
\begin{aligned}
& R B_{1}=\left\{v \in S_{B} \mid d_{G_{R B}}(v) \geq 2\right\} \\
& R B_{2}=\left\{v \in S_{B} \backslash R B_{1} \mid v \text { has a neighbor in } G_{R B} \text { that belongs to } R_{1} B\right\} \\
& R B_{3}=S_{B} \backslash\left(R B_{1} \cup R B_{2}\right) .
\end{aligned}
$$

We note that every vertex in $R_{3}$ has degree 1 in $G_{R}$, and every vertex in $R_{3} B$ has degree 1 in $G_{R B}$. Analogously, every vertex in $B_{3}$ and $R B_{3}$ has degree 1 in $G_{B}$ and $G_{R B}$, respectively. In particular, vertices from $R_{3} \cap R_{3} B$ and from $B_{3} \cap R B_{3}$ have degree 2 in $G$. Further, the neighbor of a vertex from $R_{3}$ in $G_{R}$ belongs to $R_{3}$, and, analogously, the neighbor of a vertex from $B_{3}$ in $G_{B}$ belongs to $B_{3}$. We proceed further with the following series of structural properties of the graph $G$.

Claim 4.3. $\delta(G)=2$.

Proof. Recall that $G$ is a sparse TDP-graph with associated partition $S=\left(S_{R}, S_{B}\right)$. Thus, $S_{R}$ and $S_{B}$ are disjoint total dominating sets of $G$ which form a partition of $V(G)$. Every vertex $v \in V(G)$ has at least one neighbor in $S_{R}$ and at least one neighbor in $S_{B}$. Hence, $\delta(G) \geq 2$. Suppose, to the contrary, that $\delta(G)>2$. 
Suppose that $R_{1} B \neq \emptyset$ and let $v \in R_{1} B$. Let $v_{1}, \ldots, v_{k}$, where $k \geq 2$, be the neighbors of $v$ in $G_{R B}$. By Claim 4.2 and the definition of the set $R B_{2}$, we note that for each $i \in[k]$, $v_{i} \in R B_{2}$ and the vertex $v$ is the only neighbor of $v_{i}$ that belongs to the set $S_{R}$. Further, since $d_{G}\left(v_{i}\right)>2$, the vertex $v_{i}$ has at least two neighbors in $S_{B}$. By Claim 4.2, every component of the graph $G_{B}$ is a star, implying that no two neighbors of $v$ are adjacent or have a common neighbor in $G_{B}$. Further, every neighbor of $v_{i}$ in $G$ different from $v$ belongs to the set $B_{2}$, and has the vertex $v_{i}$ as its only neighbor in $G_{B}$. Thus, the set $B_{2}$ contains at least $2 k$ vertices at distance 2 from $v$ in $G$.

For $i \in[k]$, let $w_{i}$ denote an arbitrary neighbor of $v_{i}$ in $G_{B}$, and so $w_{i} \in B_{2}$. Since $d_{G}\left(w_{i}\right)>2$ and $w_{i}$ has only one neighbor in $S_{B}$, namely the vertex $v_{i}$, we note that $w_{i} \in R B_{1}$ and therefore $w_{i}$ has at least two neighbors in $R_{2} B$. By Claim 4.2 and the definition of the set $R_{2} B$, we note that every neighbor of $w_{i}$ different from $v_{i}$ belongs to the set $R_{2} B$. Further, each such neighbor of $w_{i}$ has exactly one neighbor that belongs to the set $S_{B}$, namely the vertex $w_{i}$, and therefore has at least two neighbors in $S_{R}$ by the minimum degree condition. By Claim 4.2, every component of the graph $G_{R}$ is a star, and therefore two distinct vertices of degree at least 2 in $G_{R}$ belong to different components of $G_{R}$. This implies that this subset $R_{2} B$ of vertices in $S_{R}$ contains at least $4 k$ vertices.

By the minimum degree condition, these vertices in $R_{2} B$ also belong to $R_{1}$ and each of them has at least two neighbors in $R_{2}$. Further, analogously as before, no two such vertices are the same, implying that this subset of $R_{2}$ contains at least $8 k-1$ vertices distinct from $v$, all of which belong to the set $R_{1} B$, noting that one of these vertices may possibly be the vertex $v$. By repeating this process for all these vertices we see that we have an infinite process with infinite growth, which is not possible in a finite graph $G$. Therefore, the set $R_{1} B=\emptyset$. Analogously, the set $R B_{1}=\emptyset$. Therefore, $R_{2} B$ and $R B_{2}$ are also empty.

We now consider a vertex $v \in R_{3} B$. By Claim 4.2, every component of the graph $G_{R B}$ is a star, implying that the vertex $v$ has exactly one neighbor in $S_{B}$ and, by the minimum degree condition, at least two neighbors in $S_{R}$. Thus, $v \in R_{1}$ and each neighbor of $v$ in $S_{R}$ belong to $R_{2}$. Further, by Claim 4.2, each such neighbor of $v$ in $R_{2}$ has degree 1 in $G_{R}$ and, therefore, by the minimum degree condition, has at least two neighbors in $S_{B}$. Thus, every neighbor of $v$ in $R_{2}$ belongs to the set $R_{1} B$, contradicting our earlier observation that the set $R_{1} B$ is an empty set. This completes the proof of Claim 4.3.

By Claim 4.3, every sparse TDP-graph has minimum degree 2. In particular, $\delta(G)=2$. Let $D=\left\{v \in V(G) \mid d_{G}(v)=2\right\}$.

Claim 4.4. If a vertex in $D$ is a cut-vertex of $G$, then $(G, S) \in \mathcal{G}$.

Proof. Suppose that a vertex in $D$ is a cut-vertex of $G$. Suppose firstly that $D$ contains two adjacent vertices, $x$ and $y$, that are both cut-vertices of $G$, and let $e=x y$. Let $C_{x}$ and $C_{y}$ be the components of $G-e$ which contain $x$ and $y$, respectively. Further, let $x^{\prime}$ be the neighbor of $x$ in $C_{x}$ and let $y^{\prime}$ be the neighbor of $y$ in $C_{y}$. We have two possibilities with respect to the color of the vertices $x, x^{\prime}, y, y^{\prime}$. Either color $\left(x^{\prime}\right)=\operatorname{color}(x) \neq \operatorname{color}(y)=\operatorname{color}\left(y^{\prime}\right)$ or $\operatorname{color}\left(x^{\prime}\right)=\operatorname{color}\left(y^{\prime}\right) \neq \operatorname{color}(x)=\operatorname{color}(y)$. In both cases, let $G^{\prime}$ be the graph obtained from $G-\{x, y\}$ by adding the edge $x^{\prime} y^{\prime}$, and changing the color of all vertices in $V\left(C_{y}\right) \backslash\{y\}$ while retaining the color of all vertices in $V\left(C_{x}\right) \backslash\{x\}$. Let $S^{\prime}=\left(S_{R}^{\prime}, S_{B}^{\prime}\right)$ be the resulting partition of $V\left(G^{\prime}\right)$. We note that $G^{\prime}$ is a TDP-graph, where $S^{\prime}=\left(S_{R}^{\prime}, S_{B}^{\prime}\right)$ is a partition of $V\left(G^{\prime}\right)$ into two total dominating sets and that $x^{\prime}$ and $y^{\prime}$ are cut vertices of $G^{\prime}$. If $x$ and $x^{\prime}$ have the same color in $G$, then we use Statement 4.1 with the operation $\mathcal{O}_{17}$ 
and the cut vertex $y^{\prime}$ to show that $(G, S) \in \mathcal{G}$, while if $x$ and $x^{\prime}$ have different color in $G$, we use Statement 4.1 with the operation $\mathcal{O}_{17}$ and the cut vertex $x^{\prime}$.

Thus, we may assume that no two adjacent vertices of $D$ are both cut-vertices of $G$. Let $v$ be a cut-vertex of $G$ that belongs to $D$ with neighbors $u^{\prime}$ and $u^{\prime \prime}$. Without loss of generality we may assume that $\operatorname{color}(v)=\operatorname{color}\left(u^{\prime \prime}\right) \neq \operatorname{color}\left(u^{\prime}\right)$. Let $C_{u^{\prime}}$ and $C_{u^{\prime \prime}}$ be the components of $G-v$ containing $u^{\prime}$ and $u^{\prime \prime}$, respectively. Since $S=\left(S_{R}, S_{B}\right)$ is a partition of $V(G)$ into two total dominating sets of $G$, there exists a neighbor of $u^{\prime}$ in $C_{u^{\prime}}$ of the same color as $u^{\prime}$ and a neighbor of $u^{\prime \prime}$ in $C_{u^{\prime \prime}}$ whose color is different from that of $u^{\prime \prime}$. Let $G^{\prime}$ be the graph obtained from $G-v$ by identifying the vertices $u^{\prime}$ and $u^{\prime \prime}$ into one new vertex $u$, and joining $u$ to every neighbor of $u^{\prime}$ and $u^{\prime \prime}$. Further, we assign to $u$ the same color as that of $u^{\prime}$, while we change the color of all vertices in $V\left(C_{u^{\prime \prime}}\right) \backslash\left\{u^{\prime \prime}\right\}$ and retain the color of all vertices in $V\left(C_{u^{\prime}}\right) \backslash\left\{u^{\prime}\right\}$. Let $S^{\prime}=\left(S_{R}^{\prime}, S_{B}^{\prime}\right)$ be the resulting partition of $V\left(G^{\prime}\right)$. We note that $G^{\prime}$ is a TDP-graph, where $S^{\prime}=\left(S_{R}^{\prime}, S_{B}^{\prime}\right)$ is a partition of $V\left(G^{\prime}\right)$ into two total dominating sets. We now use Statement 4.1 with the operation $\mathcal{O}_{17}$ to show that $(G, S) \in \mathcal{G}$, where $H_{1}^{u}=C_{u^{\prime}}$ and $H_{2}^{u}=C_{u^{\prime \prime}}$.

By Claim 4.4, we may assume that no vertex in $D$ is a cut-vertex of $G$, for otherwise the desired result follows. We note that every vertex in $D$ has one neighbor in $S_{R}$ and one neighbor in $S_{B}$. Further, every component in $G[D]$ is a path or a cycle.

Claim 4.5. Let $C$ be a component of $G[D]$. If $C$ is a cycle or if $C$ is a path of order at least 5 or if $C$ is a path of order 4 and the ends of $C$ do not have a common neighbor, then $(G, S) \in \mathcal{G}$.

Proof. Suppose that $C$ is a cycle. Since $G$ is a connected TDP-graph, this implies that $G \cong C_{n}$ where $n \equiv 0(\bmod 4)$. In this case, $G$ can be obtained from the 2 -colored base graph $G_{1}$ by repeated applications of operation $\mathcal{O}_{9}$ (or operation $\mathcal{O}_{10}$ ). Hence, we may assume that $C$ is a path, for otherwise the desired result follows. Let $C$ be the path $x_{1} \ldots x_{k}$, where $k \geq 4$. Let $u$ be the neighbor of $x_{1}$ not on $C$. If $k \geq 5$, let $v=x_{5}$, while if $k=4$, let $v$ be the neighbor of $x_{4}$ not on $C$. By assumption, $u \neq v$. Let $X=\left\{x_{1}, x_{2}, x_{3}, x_{4}\right\}$.

Suppose first that $\operatorname{color}(u)=\operatorname{color}\left(x_{1}\right)$, implying that $\operatorname{color}\left(x_{2}\right)=\operatorname{color}\left(x_{3}\right) \neq$ $\operatorname{color}\left(x_{4}\right)=\operatorname{color}(v)=\operatorname{color}\left(x_{1}\right)$. If $u$ and $v$ are adjacent in $G$, let $G^{\prime}=G-X$. In this case, the graph $G^{\prime}$ is a TDP-graph and we use Statement 4.1 with the operation $\mathcal{O}_{7}$ to show that $(G, S) \in \mathcal{G}$. If $u$ and $v$ are not adjacent in $G$, let $G^{\prime}$ be obtained from $G-X$ by adding the edge $u v$. Once again, the graph $G^{\prime}$ is a TDP-graph. We use Statement 4.1 with the operation $\mathcal{O}_{10}$ to show that $(G, S) \in \mathcal{G}$.

Suppose next that $\operatorname{color}(u) \neq \operatorname{color}\left(x_{1}\right)$, implying that $\operatorname{color}\left(x_{2}\right)=\operatorname{color}(v) \neq$ $\operatorname{color}\left(x_{3}\right)=\operatorname{color}\left(x_{4}\right)=\operatorname{color}(u)$. If $u$ and $v$ are adjacent in $G$, let $G^{\prime}=G-X$. In this case, the graph $G^{\prime}$ is a TDP-graph and we use Statement 4.1 with the operation $\mathcal{O}_{8}$ to show that $(G, S) \in \mathcal{G}$. If $u$ and $v$ are not adjacent in $G$, let $G^{\prime}$ be obtained from $G-X$ by adding the edge $u v$. Once again, the graph $G^{\prime}$ is a TDP-graph. We use Statement 4.1 with the operation $\mathcal{O}_{9}$ to show that $(G, S) \in \mathcal{G}$.

By Claim 4.5, we may assume that every component of $G[D]$ is a path-component of order at most 4 , and that the ends of a path-component of $G[D]$ of order 4 have a common neighbor in $G$. In what follows we adopt the following notation. Let $P$ be a path-component of $G[D]$, and so $P \cong P_{k}$ for some $k \in[4]$. Let $P$ be the path $x_{1} \ldots x_{k}$, and let $u$ and $v$ be the vertices in $G$ that do not belong to $P$ and are adjacent to $x_{1}$ and $x_{k}$, 
respectively. We call $u$ and $v$ the vertices in $G-V(P)$ associated with the path $P$. By assumption, if $k=4$, then $u=v$. We note that if $k=1$, then $u \neq v$. We define next a good path-component.

Definition 4.6. A path-component $P$ of $G[D]$ is a good path-component if $P \cong P_{k}$ where $k \in[3]$, and both $u$ and $v$ have neighbors of both colors in the graph $G^{-}=G-V(P)$, where $u$ and $v$ are the vertices in $G^{-}$associated with $P$.

Claim 4.7. If $G[D]$ contains a good path-component, then $(G, S) \in \mathcal{G}$.

Proof. Suppose that $G[D]$ contains a good path-component, $P: x_{1} \ldots x_{k}$. By definition, $k \in[3]$. Suppose that $k=1$. Since $P$ is a good path-component, the graph $G^{\prime}=G-x_{1}$ is a TDP-graph. Furthermore, $\operatorname{color}(u) \neq \operatorname{color}(v)$ since $G$ is a TDP-graph. We now use Statement 4.1 with the operation $\mathcal{O}_{3}$ to show that $(G, S) \in \mathcal{G}$.

Suppose that $k=2$. Suppose that $\operatorname{color}\left(x_{1}\right)=\operatorname{color}\left(x_{2}\right)$. Then, $\operatorname{color}(u) \neq \operatorname{color}\left(x_{1}\right)$ and either $u=v$ or $u \neq v$ and color $(u)=\operatorname{color}(v)$. In both cases, since $P$ is a good pathcomponent, the graph $G^{\prime}=G-V(P)$ is a TDP-graph. If $u=v$, we use Statement 4.1 with the operation $\mathcal{O}_{11}$ to show that $(G, S) \in \mathcal{G}$, while if $u \neq v$, we use Statement 4.1 with the operation $\mathcal{O}_{4}$ to show that $(G, S) \in \mathcal{G}$. Suppose that $\operatorname{color}\left(x_{1}\right) \neq \operatorname{color}\left(x_{2}\right)$. Then, $\operatorname{color}(u)=\operatorname{color}\left(x_{1}\right)$ and $\operatorname{color}(v)=\operatorname{color}\left(x_{2}\right)$. Since $P$ is a good path-component, the graph $G^{\prime}=G-V(P)$ is a TDP-graph, and we use Statement 4.1 with the operation $\mathcal{O}_{5}$ to show that $(G, S) \in \mathcal{G}$.

Suppose that $k=3$. Without loss of generality we may assume that $\operatorname{color}\left(x_{1}\right) \neq$ $\operatorname{color}\left(x_{2}\right)=\operatorname{color}\left(x_{3}\right)$, implying that $\operatorname{color}(u)=\operatorname{color}\left(x_{1}\right)$ and either $u=v$ or $u \neq v$ and $\operatorname{color}(u)=\operatorname{color}(v)$. Since $P$ is a good path-component, the graph $G^{\prime}=G-V(P)$ is a TDP-graph. If $u=v$, we use Statement 4.1 with the operation $\mathcal{O}_{12}$ to show that $(G, S) \in \mathcal{G}$, while if $u \neq v$, we use Statement 4.1 with the operation $\mathcal{O}_{6}$ to show that $(G, S) \in \mathcal{G}$.

By Claim 4.7, we may assume that $G$ contains no good path-component, for otherwise the desired result follows. We define next an end-block path component of $G[D]$.

Definition 4.8. A path-component $P$ of $G[D]$ with associated vertices $u$ and $v$ is an endblock path component of $G[D]$ if $u=v$.

We are now in a position to present the following property of non-backtracking walks in the graph $G$.

Claim 4.9. Suppose that $W: w_{1} w_{2} \ldots w_{k}$ is a non-backtracking walk in $G$ and no vertex of $W$ belongs to an end-block path component of $G[D]$. If $w_{2}$ is not the only neighbor of $w_{1}$ in $G$ whose color is color $\left(w_{2}\right)$, then $w_{i-1}$ is the only neighbor of $w_{i}$ in $G$ whose color is color $\left(w_{i-1}\right)$ for all $i \in[k] \backslash\{1\}$.

Proof. Since $W$ is a non-backtracking walk in $G$, we note that no two consecutive edges on $W$ are equal; that is, $w_{i-1} \neq w_{i+1}$ for all $i \in[k-1] \backslash\{1\}$. Suppose, to the contrary, that the claim is false. Let $\ell \geq 2$ be the smallest integer such that the vertex $w_{\ell}$ has a neighbor different from $w_{\ell-1}$ of the same color as $w_{\ell-1}$.

Claim 4.9.1. $\ell \geq 3$. 
Proof. Renaming colors if necessary, we may assume that color $\left(w_{1}\right)=X$. By supposition, at least one neighbor, say $v_{1}$, of $w_{1}$ different from $w_{2}$ has the same color as $w_{2}$. Suppose firstly that color $\left(w_{2}\right)=X$. By supposition, color $\left(v_{1}\right)=X$. If $w_{2}$ has a neighbor, $z_{2}$ say, different from $w_{1}$, of color $X$, then either $v_{1}=z_{2}$, in which case $v_{1} w_{1} w_{2} v_{1}$ is a 3cycle in $G_{X}$, or $v_{1} \neq z_{2}$, in which case $v_{1} w_{1} w_{2} z_{2}$ is a path $P_{4}$ in $G_{X}$. Both cases produce a contradiction. Suppose secondly that $\operatorname{color}\left(w_{2}\right)=\bar{X}$. By supposition, $\operatorname{color}\left(v_{1}\right)=\bar{X}$. If $w_{2}$ has a neighbor, $z_{2}$ say, different from $w_{1}$, of color $X$, then $v_{1} w_{1} w_{2} z_{2}$ is a path $P_{4}$ in $G_{R B}$, a contradiction. We deduce, therefore, that $w_{1}$ is the only neighbor of $w_{2}$ whose color is $\operatorname{color}\left(w_{1}\right)$. Hence, $\ell \geq 3$.

By Claim 4.9.1, we have that $\ell \geq 3$. Renaming colors if necessary, we may assume that $\operatorname{color}\left(w_{\ell-1}\right)=X$. By supposition, the vertex $w_{\ell}$ has a neighbor, $v_{\ell+1}$ say, different from $w_{\ell-1}$ of the same color as $w_{\ell-1}$; that is, $\operatorname{color}\left(v_{\ell+1}\right)=X$. Further since $G$ is a TPD-graph, the vertex $w_{\ell}$ has a neighbor of color $\bar{X}$.

Claim 4.9.2. $d_{G}\left(w_{\ell-1}\right)=2$.

Proof. Suppose that $d_{G}\left(w_{\ell-1}\right) \geq 3$. Let $v_{\ell}$ be a neighbor of $w_{\ell-1}$ different from $w_{\ell-2}$ and $w_{\ell}$. Suppose that color $\left(w_{\ell-2}\right)=X$. By the minimality of $\ell$, the vertex $w_{\ell-2}$ is the only neighbor of $w_{\ell-1}$ whose color is color $\left(w_{\ell-2}\right)$; that is, all neighbors of $w_{\ell-1}$ different from $w_{\ell-2}$ must have color $\bar{X}$. In particular, $\operatorname{color}\left(v_{\ell}\right)=\operatorname{color}\left(w_{\ell}\right)=\bar{X}$. Hence, $v_{\ell} w_{\ell-1} w_{\ell} v_{\ell+1}$ is a path $P_{4}$ in $G_{R B}$, a contradiction. Hence, $\operatorname{color}\left(w_{\ell-2}\right)=\bar{X}$. Thus, all neighbors of $w_{\ell-1}$ different from $w_{\ell-2}$ must have color $X$. In particular, $\operatorname{color}\left(v_{\ell}\right)=$ $\operatorname{color}\left(w_{\ell}\right)=X$. If $v_{\ell}=v_{\ell+1}$, then $v_{\ell} w_{\ell-1} w_{\ell} v_{\ell}$ is a 3 -cycle in $G_{X}$, a contradiction. If $v_{\ell} \neq v_{\ell+1}$, then $v_{\ell} w_{\ell-1} w_{\ell} v_{\ell+1}$ is a path $P_{4}$ in $G_{X}$, a contradiction.

By Claim 4.9.2, the vertex $w_{\ell-1}$ has degree 2 in $G$; that is, $w_{\ell-1} \in D$. By supposition, the vertex $w_{1}$ has at least two neighbors whose color is color $\left(w_{2}\right)$ and at least one vertex whose color is different from color $\left(w_{2}\right)$. In particular, the vertex $w_{1}$ has degree at least 3 in $G$. Let $p \geq 1$ be the largest integer such that $d_{G}\left(w_{p}\right) \geq 3$ and $p \leq \ell-2$. Possibly, $p=\ell-2$. We now consider the path $P: w_{p+1} \ldots w_{\ell-1}$ and note that $P$ is a path-component in $G[D]$. If $w_{p}=w_{\ell}$, then $P$ is an end-block path component of $G[D]$, contradicting the supposition that no vertex of $W$ belongs to an end-block path component of $G[D]$. Hence, $w_{p} \neq w_{\ell}$ and the vertices $w_{p}$ and $w_{\ell}$ associated with the path-component $P$ in $G[D]$ are distinct vertices.

We now consider the graph $G^{-}=G-V(P)$. By our earlier observations, the vertex $w_{\ell}$ has neighbors of both colors in $G^{-}$. If $p=1$, then the vertex $w_{p}$ has neighbors of both colors in $G^{-}$. If $p \geq 2$, then by the minimality of $\ell$ the vertex $w_{p}$ once again has neighbors of both colors in $G^{-}$. Thus the path $P$ is a good-path component, contradicting our earlier assumption that $G$ contains no good path-component. This completes the proof of Claim 4.9.

Claim 4.10. If $G$ contains a cycle that is not an end-block of $G$, then $(G, S) \in \mathcal{G}$.

Proof. Assume that some cycle $C$ in $G$ is not an end-block in $G$. Let $P$ be a pathcomponent of $G[D]$ with associated vertices $u$ and $v$. Suppose firstly that $u=v$. Thus, $P$ is an end-block path component of $G[D]$ and $C_{P}=G[V(P) \cup\{u\}]$ is a cycle in $G$. Further, $C_{P}$ is an end-block of $G$ with $u$ as its cut-vertex in $G$. Suppose that $d_{G}(u) \geq 4$. We now consider the graph $G^{-}=G-V(P)$. By our earlier assumptions, no vertex in $D$ is a cut-vertex of $G$, implying that $G^{-}$is a connected graph. 
Claim 4.10.1. The vertex $u$ has neighbors of both colors in $G^{-}$.

Proof. Suppose, to the contrary, that all neighbors of $u$ in $G^{-}$have the same color. By supposition, there is a cycle $C$ in $G^{-}$that contains no vertex that belongs to an end-block component of $G[D]$. Hence there exists a non-backtracking walk $W: w_{1} w_{2} \ldots w_{k}$ in $G$ that starts at the vertex $u$, proceeds from $u$ to $C$, goes all the way around $C$, and then returns to $u$, without entering any end-block path component of $G[D]$. We note that $k \geq 3$ and that $w_{1}=w_{k}=u$. By our supposition that all neighbors of $u$ in $G^{-}$have the same color, the vertex $w_{2}$ is not the only neighbor of $w_{1}$ in $G$ whose color is color $\left(w_{2}\right)$. By Claim 4.9, the vertex $w_{k-1}$ is the only neighbor of $w_{k}$ in $G$ whose color is color $\left(w_{k-1}\right)$. This contradicts our supposition that all neighbors of $u$ in $G^{-}$have the same color.

By Claim 4.10.1, the vertex $u$ has neighbors of both colors in $G^{-}$. Since $G$ is a TDPgraph, this implies that the graph $G^{-}$is a TDP-graph. Hence, we can use Statement 4.1 with the operation $\mathcal{O}_{11}$ or $\mathcal{O}_{12}$ or $\mathcal{O}_{13}$, depending on the length of $P$, to show that $(G, S) \in \mathcal{G}$. We may therefore assume that $d_{G}(u)=3$ (and still $u=v$ ), for otherwise $(G, S) \in \mathcal{G}$, as desired. Thus, the vertex $u$ has degree 1 in $G^{-}$. Let $x$ be the neighbor of $u$ in $G^{-}$. By our earlier assumptions, no vertex in $D$ is a cut-vertex of $G$. In particular, the cutvertex $x$ does not belong to $D$, and so $d_{G}(x) \geq 3$. We now consider the (connected) graph $G_{u}^{-}=G^{-}-u$ obtained from $G^{-}$by deleting the vertex $u$. Using analogous arguments as in the proof of Claim 4.10.1, the vertex $x$ has neighbors of both colors in $G_{u}^{-}$. Hence, we can use Statement 4.1 with the operation $\mathcal{O}_{14}$ or $\mathcal{O}_{15}$ or $\mathcal{O}_{16}$, depending on the length of $P$, to show that $(G, S) \in \mathcal{G}$.

Suppose next that $u \neq v$. Using analogous arguments as in the proof of Claim 4.10.1, the vertices $u$ and $v$ each have neighbors of both colors in $G_{u}^{-}$. Thus the path $P$ is a good-path component, contradicting our earlier assumption that $G$ contains no good pathcomponent. This completes the proof of Claim 4.10.

By Claim 4.10, we may assume that every cycle in $G$ is an end-block of $G$, for otherwise $(G, S) \in \mathcal{G}$ as desired. Every block of $G$ that is not an end-block is a copy of $K_{2}$ consisting of a single edge. By our earlier assumptions, every cycle in $G$ has length 3,4 or 5 . Let $T^{-}$be the graph obtained from $G$ by deleting all vertices that belong to an end-block path component of $G[D]$. By our earlier assumptions, the graph $T^{-}$is a tree. In particular, every vertex of $T^{-}$is a cut-vertex of $G$. By our earlier assumptions, no vertex in $D$ is a cut-vertex of $G$, implying that every vertex of $D$ belongs to an end-block path component of $G[D]$. Hence, every vertex of $D$ belongs to an end-block of $G$.

Claim 4.11. If two cycles of $G$ intersect, then $(G, S) \in \mathcal{G}$.

Proof. Suppose that two different cycles $C_{1}$ and $C_{2}$ of $G$ intersect. Since every cycle in $G$ is an end-block of $G$, these two cycles intersect in exactly one common vertex, $v$ say.

Claim 4.11.1. If $G$ contains exactly one cut-vertex, then $(G, S)$ is a 2-colored base graph $G_{3}$.

Proof. Suppose that $G$ contains exactly one cut-vertex. Since the cut-vertices of $G$ are precisely the vertices in the tree $T^{-}$, this implies that $V\left(T^{-}\right)=\{v\}$. Thus, every block of $G$ is an end-block that contains the vertex $u$. Let $C_{1}$ be the cycle $v v_{1} v_{2} \ldots v_{k-1} v$ and let $\operatorname{color}(v)=X$, where $k \in\{3,4,5\}$. If $k=3$, then $\operatorname{color}\left(v_{1}\right)=\operatorname{color}\left(v_{2}\right)=\bar{X}$. If $k=4$, then $\operatorname{color}\left(v_{2}\right)=\bar{X}$ and, renaming $v_{1}$ and $v_{3}$, if necessary, we may assume that 
$\operatorname{color}\left(v_{1}\right)=X$ and $\operatorname{color}\left(v_{3}\right)=\bar{X}$. If $k=5$, then $\operatorname{color}\left(v_{2}\right)=\operatorname{color}\left(v_{3}\right)=\bar{X}$ and $\operatorname{color}\left(v_{1}\right)=\operatorname{color}\left(v_{4}\right)=X$.

Suppose that $G$ contains an end-block, $C$ say, that is a 4-cycle. If $C^{\prime}$ is an arbitrary endblock different from $C$, then $C^{\prime}-v$ is a good path-component of $G[D]$, a contradiction. Hence, no end-block of $G$ is a 4-cycle.

Thus, since $G$ is a TDP-graph, at least one end-block is a 3-cycle and at least one endblock is a 5-cycle. Renaming the end-blocks if necessary, we may assume that $C_{1}$ is a 3 -cycle and $C_{2}$ is a 5 -cycle. These two cycles, together with their associated 2-colorings described above, form the 2-colored base graph $G_{3}$. If $G$ contains at least three blocks and $C^{\prime}$ is an arbitrary end-block different from $C_{1}$ and $C_{2}$, then $C^{\prime}-v$ is a good pathcomponent of $G[D]$, a contradiction. Hence, $G$ contains exactly two end-blocks, implying that $(G, S)$ is the 2 -colored base graph $G_{3}$.

By Claim 4.11.1, we may assume that $G$ contains at least two cut-vertices, for otherwise $(G, S) \in \mathcal{G}$ as desired. As observed earlier, the cut-vertices of $G$ are precisely the vertices in the tree $T^{-}$. Let $x$ be a neighbor of $v$ in $T^{-}$. Renaming the cycle $C_{1}$ and $C_{2}$ and the vertex $x$ if necessary, we may assume without loss of generality that the vertex $v$ has a neighbor, $y$ say, in $C_{1}$ such that $\operatorname{color}(x) \neq \operatorname{color}(y)$. We now consider the graph $G^{-}=$ $G-\left(V\left(C_{2}\right) \backslash\{v\}\right)$. Since $G$ is a TDP-graph, this implies that the graph $G^{-}$is a TDP-graph. Hence, we can use Statement 4.1 with the operation $\mathcal{O}_{11}$ or $\mathcal{O}_{12}$ or $\mathcal{O}_{13}$, depending on the length of $C_{2}$, to show that $(G, S) \in \mathcal{G}$.

By Claim 4.11, we may assume that no two cycles of $G$ intersect, for otherwise $(G, S) \in$ $\mathcal{G}$ as desired. The tree $T^{-}$therefore contains at least two vertices. Further, every leaf in $T^{-}$ has degree 3 in $G$ and belongs to exactly one end-block of $G$. Let $p_{1} p_{2} \ldots p_{k}$ be a longest path in $T^{-}$. Necessarily, $p_{1}$ and $p_{k}$ are both leaves in $T^{-}$. Since $T^{-}$contains no vertex of $D$, we note that every vertex in $T^{-}$has degree at least 3 in $G$. Let $C_{1}$ and $C_{k}$ be the end-blocks in $G$ that contain $p_{1}$ and $p_{k}$, respectively.

Claim 4.12. If $k \in\{2,3\}$, then $(G, S) \in \mathcal{G}$.

Proof. Suppose firstly that $k=2$. In this case, $G$ is obtained from the two cycles $C_{1}$ and $C_{2}$ by adding the edge $p_{1} p_{2}$. If $C_{1}$ is a 4 -cycle, then the cycle $C_{1}$ together with its associated 2-coloring is the 2-colored base graph $G_{1}$. Starting with this 2-colored base graph $G_{1}$, we can use Statement 4.1 with the operation $\mathcal{O}_{14}$ or $\mathcal{O}_{15}$ or $\mathcal{O}_{16}$, depending on the length of $C_{2}$, to show that $(G, S) \in \mathcal{G}$. Analogously, if $C_{2}$ is a 4-cycle, $(G, S) \in \mathcal{G}$. Hence, we may assume that neither $C_{1}$ nor $C_{2}$ is a 4-cycle. With this assumption, if $C_{1}$ is a 3 -cycle, then $C_{2}$ is also a 3 -cycle noting that $G$ is a TDP-graph. In this case, $(G, S)$ is the 2 -colored base graph $G_{2}$. If $C_{1}$ is a 5 -cycle, then $C_{2}$ is also a 5 -cycle. In this case, $(G, S)$ is the 2-colored base graph $G_{4}$. Hence if $k=2$, then $(G, S) \in \mathcal{G}$.

Suppose secondly that $k=3$. We now consider the (connected) graph $G^{-}=G-$ $V\left(C_{1}\right)$. We note that the vertex $p_{2}$ has degree at least 2 in $G^{-}$. If the vertex $p_{2}$ has neighbors of both colors in $G^{-}$, then $G^{-}$is a TPD-graph. In this case, we can use Statement 4.1 with the operation $\mathcal{O}_{14}$ or $\mathcal{O}_{15}$ or $\mathcal{O}_{16}$, depending on the length of $C_{1}$, to show that $(G, S) \in \mathcal{G}$. Hence we may assume that all neighbors of $p_{2}$ in $G^{-}$have the same color which is different to color $\left(p_{1}\right)$ (noting that $G$ is a TPD-graph). This implies that the vertex $p_{2}$ has neighbors of both colors in the graph $G-V\left(C_{2}\right)$, and once again we can use Statement 4.1 with the operation $\mathcal{O}_{14}$ or $\mathcal{O}_{15}$ or $\mathcal{O}_{16}$, depending on the length of $C_{2}$, to show that $(G, S) \in \mathcal{G}$. 
By Claim 4.12, we may assume that $k \geq 4$, for otherwise $(G, S) \in \mathcal{G}$ as desired. We now consider the (connected) graph $G^{-}=G-V\left(C_{1}\right)$. If the vertex $p_{2}$ has neighbors of both colors in $G^{-}$, then as in the proof of Claim 4.12 we can use Statement 4.1 with the operation $\mathcal{O}_{14}$ or $\mathcal{O}_{15}$ or $\mathcal{O}_{16}$, depending on the length of $C_{1}$, to show that $(G, S) \in \mathcal{G}$. Hence we may assume that all neighbors of $p_{2}$ in $G^{-}$have the same color. We now consider the walk $p_{2} p_{3} \ldots p_{k}$. By assumption, $p_{3}$ is not the only neighbor of $p_{2}$ in $G$ whose color is color $\left(p_{3}\right)$. By Claim 4.9, the vertex $p_{k-2}$ is the only neighbor of $p_{k-1}$ in $G$ whose color is color $\left(p_{k-2}\right)$. This implies that the vertex $p_{k-1}$ has neighbors of both colors in the graph $G-V\left(C_{2}\right)$. Hence, $G-V\left(C_{2}\right)$ is a TPD-graph and we can use Statement 4.1 with the operation $\mathcal{O}_{14}$ or $\mathcal{O}_{15}$ or $\mathcal{O}_{16}$, depending on the length of $C_{2}$, to show that $(G, S) \in \mathcal{G}$. This completes the proof of Theorem 3.1.

\section{Closing remarks}

We remark that although our characterization in Theorem 3.1 solves a long-standing problem in the theory of total domination in graphs which has been open for several decades, it remains a challenging problem to determine in polynomial time if a given graph is a TDP-graph even for some special graph classes. Our method cannot be used to decide if a given graph $G$ is a TDP-graph in polynomial time. The reason for that is that we have no specified vertex partition together with $G$. Indeed, recognizing this class of graphs is known to be NP-complete (see [8]). However, we nonetheless believe that our constructive proof gives valuable insights into the problem and gives an entirely new description of TDP-graphs, placing them in another context.

We close with a short discussion about the independence of operations $\mathcal{O}_{1}$ to $\mathcal{O}_{17}$ in the class $\mathcal{G}$. For this purpose, we will construct small graphs in $\mathcal{G}$ from our 2-colored base graphs that cannot be built by any other construction in $\mathcal{G}$, thereby showing that operation $\mathcal{O}_{i}$ is independent for each $i \in[17]$. The independence of these seventeen operations used to build graphs in the family $\mathcal{G}$ show that none of them are redundant, and all are needed in the construction.

- Apply operation $\mathcal{O}_{2}$ on $G_{1}$ (to obtain the graph $K_{4}-e$ ).

- Apply operation $\mathcal{O}_{3}$ on $G_{1}$ to obtain the house graph; that is, the graph obtained from a 5 -cycle by adding an edge.

- Apply operation $\mathcal{O}_{1}$ once and operation $\mathcal{O}_{2}$ three times on the house graph to obtain $K_{5}$.

- Apply operation $\mathcal{O}_{4}$ to two nonadjacent vertices of degree 2 on $G_{2}$.

- The independence of operation $\mathcal{O}_{x}$, where $x \in\{5,6,11,12,13,14,15,16\}$, can be seen by applying $\mathcal{O}_{x}$ once on $G_{1}$.

- The independence of operation $\mathcal{O}_{x}$, where $x \in\{7,10\}$, can be seen by applying $\mathcal{O}_{x}$ once on adjacent vertices of degree 3 in $G_{2}$.

- The independence of operation $\mathcal{O}_{x}$, where $x \in\{8,9\}$, can be seen by applying $\mathcal{O}_{x}$ once on adjacent vertices of degree 3 in $G_{4}$.

- Apply operation $\mathcal{O}_{17}$ once on the cut-vertex of $G_{3}$.

Hence, all seventeen operations are independent. Further, our proof of Theorem 3.1 shows that all seventeen operations are necessary to give our characterization of TDPgraphs. 


\section{References}

[1] H. Aram, S. M. Sheikholeslami and L. Volkmann, On the total domatic number of regular graphs, Trans. Comb. 1 (2012), 45-51, doi:10.22108/toc.2012.760.

[2] B. Chen, J. H. Kim, M. Tait and J. Verstraete, On coupon colorings of graphs, Discrete Appl. Math. 193 (2015), 94-101, doi:10.1016/j.dam.2015.04.026.

[3] E. J. Cockayne, R. M. Dawes and S. T. Hedetniemi, Total domination in graphs, Networks 10 (1980), 211-219, doi:10.1002/net.3230100304.

[4] P. Delgado, W. J. Desormeaux and T. W. Haynes, Partitioning the vertices of a graph into two total dominating sets, Quaest. Math. 39 (2016), 863-873, doi:10.2989/16073606.2016. 1188862 .

[5] W. J. Desormeaux, T. W. Haynes and M. A. Henning, Partitioning the vertices of a cubic graph into two total dominating sets, Discrete Appl. Math. 223 (2017), 52-63, doi:10.1016/j.dam. 2017.01.032.

[6] T. W. Haynes, S. T. Hedetniemi and P. J. Slater (eds.), Domination in Graphs: Advanced Topics, volume 209 of Monographs and Textbooks in Pure and Applied Mathematics, Marcel Dekker, New York, 1998.

[7] T. W. Haynes, S. T. Hedetniemi and P. J. Slater, Fundamentals of Domination in Graphs, volume 208 of Monographs and Textbooks in Pure and Applied Mathematics, Marcel Dekker, New York, 1998.

[8] P. Heggernes and J. A. Telle, Partitioning graphs into generalized dominating sets, Nordic J. Comput. 5 (1998), 128-142.

[9] M. A. Henning, A survey of selected recent results on total domination in graphs, Discrete Math. 309 (2009), 32-63, doi:10.1016/j.disc.2007.12.044.

[10] M. A. Henning, C. Löwenstein, D. Rautenbach and J. Southey, Disjoint dominating and total dominating sets in graphs, Discrete Appl. Math. 158 (2010), 1615-1623, doi:10.1016/j.dam. 2010.06.004.

[11] M. A. Henning and J. Southey, A note on graphs with disjoint dominating and total dominating sets, Ars Combin. 89 (2008), 159-162.

[12] M. A. Henning and J. Southey, A characterization of graphs with disjoint dominating and total dominating sets, Quaest. Math. 32 (2009), 119-129, doi:10.2989/qm.2009.32.1.10.712.

[13] M. A. Henning and A. Yeo, Total Domination in Graphs, Springer Monographs in Mathematics, Springer, New York, 2013, doi:10.1007/978-1-4614-6525-6.

[14] O. Ore, Theory of Graphs, volume 38 of American Mathematical Society Colloquium Publications, American Mathematical Society, Providence, Rhode Island, 1962.

[15] J. Southey and M. A. Henning, A characterization of graphs with disjoint dominating and paired-dominating sets, J. Comb. Optim. 22 (2011), 217-234, doi:10.1007/ s10878-009-9274-1.

[16] B. Zelinka, Total domatic number and degrees of vertices of a graph, Math. Slovaca 39 (1989), 7-11. 